\section{Management of pneumothorax with oxygen therapy: a case series}

\author{
Amit Panjwani \\ Salmaniya Medical Complex, Manama, \\ Bahrain
}

\begin{abstract}
Pneumothorax is relatively common condition affecting individuals of all ages. Goals of treatment of pneumothorax are removal of air from the pleural cavity and prevent its recurrence. The different strategies available at the disposal of the treating physician include observation, supplemental oxygen administration, needle aspiration, insertion of small bore chest drains and last but definitely not the least, surgery in recurrent cases. Oxygen therapy is useful in the treatment of all types of pneumothorax where it is found to be safe, effective and associated with reduced length of hospital stay. Four cases of pneumothoraces are presented here which were successfully managed with supplemental oxygen administration only.
\end{abstract}

\section{Introduction}

Pneumothorax is the presence of air or gas between the parietal and visceral pleural surfaces. It may present with minimal symptoms like mild chest pain and dyspnea, which may go unnoticed by the patient for a few days, on one hand and life threatening cardiorespiratory collapse requiring immediate intervention, on the other. The treatment of pneumothorax depends on the degree of cardiorespiratory impairment, severity of symptoms and size of the pneumothorax. Treatment strategies include observation, oxygen therapy, needle aspiration or intercostal chest tube (ICT) insertion. ${ }^{1}$ Oxygen therapy is useful in the treatment of traumatic ${ }^{2}$ and spontaneous pneumothorax $^{3}$ where it is found to be safe, efficacious and associated with reduced length of hospital stay. Hospitalised cases of pneumothorax who are not subjected to needle aspiration and ICT drainage, due to any reason, may be managed with supplemental oxygen therapy administered at high concentrations. Four cases of pneumothorax that were successfully managed with oxygen therapy are presented here.

\section{Case Report \\ Case \#1}

A 19-year-old male, non-smoker, presented with sudden onset left sided chest pain and dyspnea MRC 3. There was no fever, wheeze, hemoptysis or cough. Patient denied any history of palpitations, syncope or swelling of the limbs. These symptoms reduced in intensity over the next few days. He decided to visit a general practioner (GP) for persistence of symptoms. He was evaluated by the GP and referred to emergency department of our hospital. On evaluation, the chest pain and dyspnea had significantly reduced in intensity, he was afebrile, pulse- $82 / \mathrm{min}$, respiratory rate (RR) $-20 / \mathrm{min}, \mathrm{SpO}_{2}-98 \%$ on room air and blood pressure (BP)-110/70 mm Hg. He was of average built and height. General and systemic examination was unremarkable except for reduced breath sounds heard in the entire left hemithorax. Chest radiograph (Figure 1) showed a left sided pneumothorax. The size of the pneumothorax estimated by the Rhea method ${ }^{4}$ was found to be $22 \%$ of the pleural cavity. He was admitted in the hospital and complete blood counts with biochemical profile were found to be normal. Patient was managed with oxygen administration and close monitoring for any signs of worsening of pneumothorax. Oxygen was administered through the simple face mask with the oxygen flow of $10 \mathrm{~L} /$ minute. The mask was worn continuously, except during the meal times and visiting times. He showed a gradual clinical and radiological improvement. The complete re-expansion of left lung was seen in 3 days (Figure 2) and the patient was discharged from the hospital.

\section{Case \#2}

A 23-year-old male, non smoker, no history of addictions, had a fall from his motorcycle in the early hours of the morning. He was immediately shifted to the emergency department of our hospital. The patient complained of severe right sided chest pain and dyspnea MRC 4. There was no cough, hemoptysis, fever or noisy breathing. There was no major injury to any other part of the body. On examination, he was an obese individual who was afebrile, pulse- $85 / \mathrm{min}$, RR-24/min, $\mathrm{SpO}_{2}-96 \%$ on room air and BP-120/80 mm Hg. He was conscious, oriented to time, place and person. Respiratory examination revealed tenderness over right side of the chest and reduced breath sounds in right infraclavicular and mammary areas. Otherwise the systemic examination was unremarkable. Complete blood counts with biochemical profile were found to be normal. Chest radi-
Correspondence: Amit Panjwani, Salmaniya Medical Complex, Manama, Bahrain.

E-mail: drameetp@gmail.com

Key words: Pneumothorax, Spontaneous, Traumatic, Iatrogenic, Oxygen therapy.

Received for publication: 7 September 2016 Revision received: 24 May 2017.

Accepted for publication: 25 May 2017

This work is licensed under a Creative Commons Attribution NonCommercial 4.0 License (CC BY-NC 4.0).

(C) Copyright A. Panjwani, 2017

Licensee PAGEPress, Italy

Chest Disease Reports 2017; 5:6276

doi:10.4081/cdr.2017.6276

ograph (Figure 3) showed a right sided pneumothorax with no mediastinal shift. The size of the pneumothorax estimated by the Rhea method was found to be $26 \%$ of the pleural cavity. The computed tomography $(\mathrm{CT})$ of the chest revealed undisplaced fractures involving posterior right $6^{\text {th }}, 7^{\text {th }}, 8^{\text {th }}$ and $9^{\text {th }}$ ribs. Moderate right sided pneumothorax and minimal pneumomediastinum was also seen (Figure 4). CT scan of the brain and cervical spine was found to be normal. Arterial blood gas analysis and electrocardiogram was normal. He was immediately initiated on parenteral analgesics and supplemental oxygen therapy. He was offered insertion of the intercostal tube drainage for the treatment of pneumothorax, which the patient refused. The patient was closely monitored for signs of tension pneumothorax. Oxygen was administered through the mask with the flow of $10 \mathrm{~L} / \mathrm{min}$. He gradually improved and over a period of 4 days there was a complete lung re-expansion (Figure 5).

\section{Case \#3}

A 61-year-old male, non-smoker, had undergone an open nephrectomy under general anaesthesia as a donor for renal transplantation. He developed low grade fever and cough with purulent expectoration on $3^{\text {rd }}$ post-operative day. He had no chest pain, dyspnea, wheeze or hemoptysis. He had no significant co-morbidities. On examination, temperature- $100^{\circ} \mathrm{F}$, pulse- $110 / \mathrm{min}, \mathrm{RR}$ 20/min, BP-130/80 mm Hg and $\mathrm{SpO}_{2}-97 \%$ on room air. There were reduced breath sounds in right susprascapular area with no other abnormalities seen on systemic examination. The complete blood counts and biochemical profile were unremarkable. Chest radiograph showed a loculated right apical pneumothorax with collapse of the right 
upper lobe. He was managed with parenteral antibiotics, mucolytics, chest physiotherapy and supplemental oxygen administration with a flow of $10 \mathrm{~L} / \mathrm{min}$ delivered through the simple face mask. He showed a remarkable improvement with a complete right lung re-expansion seen over the next two days.

\section{Case \#4}

A 31-year-old lady, non smoker, presented to the out-patient department of our hospital with exertional dyspnea since 1 month and central non radiating chest pain since 1 week. On evaluation she was found to have an anterior mediastinal mass for which she was subjected to a CT-guided needle biopsy. Post-procedure check CT of the thorax showed a minimal pneumothorax on right side. Patient had no increase in the intensity of symptoms. She was admitted in the hospital for observation. On examination, she was afebrile, pulse- $82 / \mathrm{min}, \mathrm{RR}$ $20 / \mathrm{min}, \mathrm{SpO}_{2}-98 \%$ on room air and BP$110 / 70 \mathrm{~mm} \mathrm{Hg}$. General and systemic examination was normal. She was treated with supplemental oxygen administration delivered through a simple face mask with a flow of $10 \mathrm{~L} / \mathrm{min}$. There was a complete resolution of the pneumothorax noted by the next day and discharged from the hospital.

\section{Discussion}

Pneumothorax is a relatively common clinical condition which affects individuals of all ages. It may be classified as primary, secondary, iatrogenic or traumatic, based on the underlying cause. ${ }^{1}$ In the present case series, there were two cases of iatrogenic pneumothorax and one each of traumatic and primary spontaneous pneumothorax. Treatment goals of pneumothorax are removal of air from the pleural cavity and prevent its recurrence. ${ }^{5}$ The different strategies available at the disposal of the treating physician are dependent on the clinical presentation of the patient. Active intervention is recommended in patient presenting with significant breathlessness associated with any size of the pneumothorax. Needle aspiration and small bore ICT are the usual options in these situations. Patients with small pneumothorax (usually less than 15\% of the hemithorax) or those with large ones with minimal symptoms can be managed with observation and administration of supplemental oxygen. Estimating the size of pneumothorax is traditionally done with help of Light's Index which utilizes the diameters of the lung and the hemithorax to arrive at the conclusion. ${ }^{6}$ Other methods to estimate the size of pneumothorax includes the Rhea method ${ }^{4}$ and the Collins method $^{7}$ which utilize a nomogram based on the average of the interpleural distances measured at the apex, midpoint of the upper part of the lung and midpoint of the lower half of the lung on an erect chest radiograph. Both these methods are good for estimating the size of small pneumothoraces however, Collins scores over the Rhea method in accurately estimating the size of larger pneumothorax. ${ }^{8}$ Alternatively, an easier way to differentiate between a large and small pneumothorax is the presence of a visible rim of air more than $2 \mathrm{~cm}$. between the lung margin and chest wall at the level of the hilum. ${ }^{9}$

Supplemental oxygen is recommended as a treatment option in all types of pneumothoraces. ${ }^{2,9}$ The rate of re-absorption of air in the pneumothorax is $1.25-1.8 \%$ of the

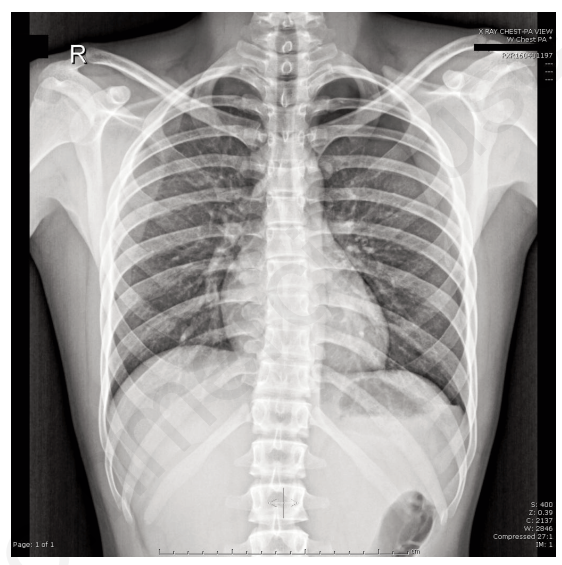

Figure 1. At the time of admission. Chest radiograph shows a left sided pneumothorax with a central mediastinum.

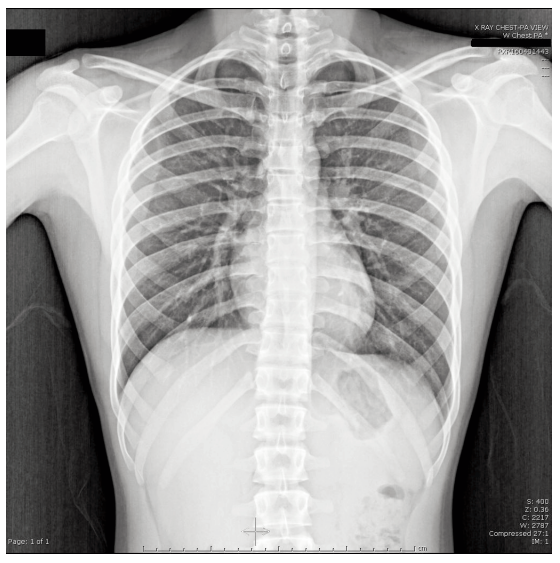

Figure 2. Day 3 after admission. Follow up chest radiograph showing a complete reexpansion of the left lung.

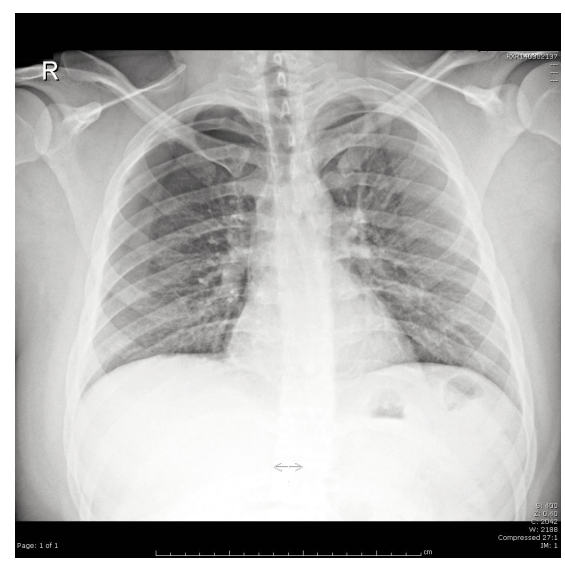

Figure 3. At the time of admission. Chest radiograph showing a right sided pneumothorax.

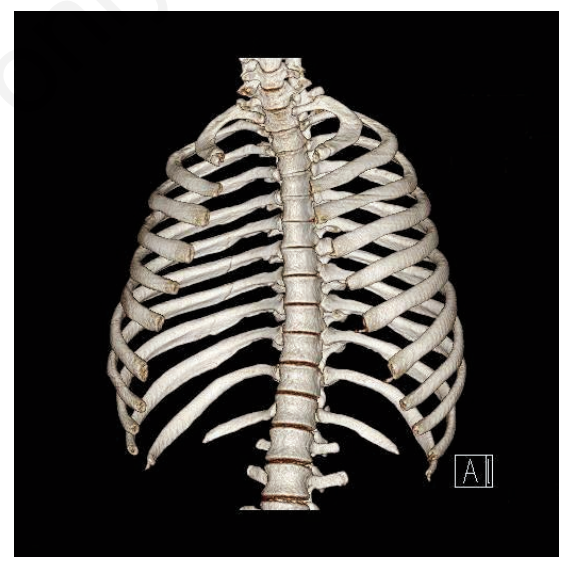

Figure 4. Computed tomography volumetric rendering technique-shaded surface display showing undisplaced fractures involving posterior right $6^{\text {th }}, 7^{\text {th }}, 8^{\text {th }}$ and $9^{\text {th }}$ ribs.

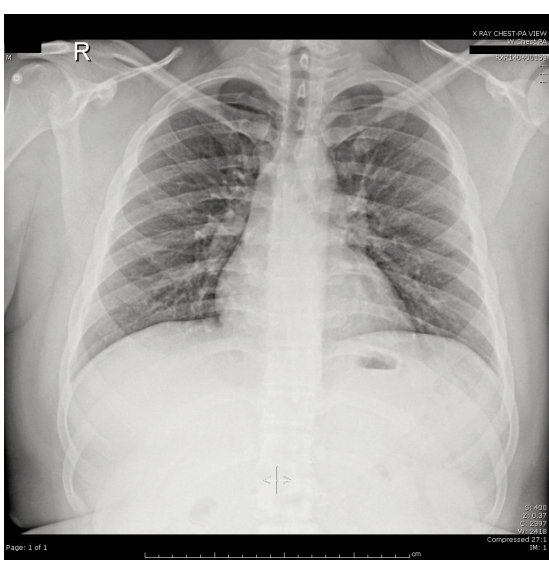

Figure 5. Day 4 after admission. Chest radiograph showing a complete resolution of pneumothorax on right side. 
volume of hemithorax every 24 hours. There is a four-fold increase in this rate of re-absorption of air with the administration of supplemental oxygen. ${ }^{10}$ In pneumothorax, the gases move in and out of the pleural space from the capillaries in the visceral and parietal pleura. The movement of each gas depends upon the gradient between its partial pressure in the capillaries and the pleural space, amount of blood flow and the solubility of each gas in the surrounding tissue. ${ }^{11}$ The partial pressure of all gases, on room air, in the capillaries of the pleural space is around $706 \mathrm{~mm} \mathrm{Hg}$. Administration of $100 \%$ oxygen causes denitrogenation of blood. The $\mathrm{PN}_{2}$ in the blood in the pleural capillaries approaches zero while the $\mathrm{PO}_{2}$ in fully oxygenated blood is about $100 \mathrm{~mm}$ $\mathrm{Hg}$, the total gas pressure in the blood therefore becomes significantly less than that in the pleural space $(760 \mathrm{~mm} \mathrm{Hg}$ in the pleural cavity and $146 \mathrm{~mm} \mathrm{Hg}$ in the pleural capillary blood when $100 \% \mathrm{O}_{2}$ is administered). This high gradient of pressure between the two adjoining surfaces increases the rate of absorption of air by several fold. ${ }^{3,12}$ In the landmark study by Northfield, treatment of pneumothorax by observation was compared with supplemental oxygen administration. The rate of absorption of gas from the pneumothorax in the oxygen therapy group was four times that of the observation group. The full re-expansion of the lung was achieved over 5 days in most of the patients. ${ }^{3}$ Similar results were seen in the present series. Usually pneumothoraces less than $30 \%$ of the hemithorax have a successful outcome with supplemental oxygen administration compared to those with larger than $30 \%$ who ultimately require intercostal tube drainage. ${ }^{13}$ All the patients in the present case series had a pneumothorax less than $30 \%$ of the pleural cavity and had a positive outcome with supplemental oxygen administration. In the experimental studies on rabbit model, there was a clear advantage demonstrated in the oxygen treated group with fraction of inspired oxygen $\left(\mathrm{FiO}_{2}\right)$ of 0.6 compared to the controls, in terms of the rate of resolution of the pneumothorax. ${ }^{14}$ Hyperoxia $\left(\mathrm{FiO}_{2}\right.$ of $\left.0.6-1.0\right)$ treatment shortens the resolution time of pneumothorax. ${ }^{15,16}$ Smaller $\mathrm{FiO}_{2}(0.3,0.4$, $0.5)$ may also be useful compared to no oxygen administration in faster improvement in patients with pneumothorax. ${ }^{17}$ In the present case series, the $\mathrm{FiO}_{2}$ was not measured but it was probably around 0.5 to 0.6 with the oxygen flow of around $10 \mathrm{~L} / \mathrm{min}$ using a simple face mask. Treatment with $\mathrm{FiO}_{2}$ of 0.6 and above for short durations is not associated with hyperoxia associated lung toxicity. ${ }^{15}$ In a few studies in the pediatric population, supple- mental oygen use was not associated with a faster resolution of spontaneous pneumothorax. ${ }^{18,19}$ Occasionally we may encounter asymptomatic patients with pneumothorax of large size refusing placement of ICT and surgery. These patients cannot be sent home without treatment. They need to be observed in the hospital for worsening of pneumothorax. During the observation period, these patients may be subjected to oxygen administration and it has been found to be useful in most of the situations. Oxygen administration as a sole treatment modality is used more often in patients with primary spontaneous pneumothorax when compared to secondary pneumothorax..$^{20}$ Care should be taken during oxygen administration to patients with COPD and pneumothorax, as they may have worsening of carbon dioxide levels.

ICT drainage is usually required in patients with tension pneumothorax, mechanically ventilated patients with pneumothorax and in those with persistent or recurrent pneumothorax. It may also be indicated in patients with pneumothorax of any size causing respiratory distress. ${ }^{21}$ The insertion of ICT helps in faster removal of air from the pleural cavity. It is associated with hospital stay of 5-7 days. ${ }^{22}$ It may be associated with pain, anxiety, infections, drain dislodgement and blockage. Visceral injury during the ICT insertion is the most serious complication. ${ }^{23}$ Its insertion requires appropriate training and supervision by experienced trainers. Oxygen therapy on the other hand, is safe, easily available, non invasive, requires no special training and has hardly any complications associated with its use.

Oxygen therapy, therefore, may be recommended in patients with pneumothorax having no significant symptoms, size usually less than $30 \%$ of the hemithorax and in those refusing active intervention. It should not be used as a sole therapy in patients with tension pneumothorax or a bilateral pneumothorax. If the supplemental oxygen administration fails, as evidenced by increase in the size of the pneumothorax or the patients developing worsening of symptoms, then ICT insertion is recommended. Supplemental oxygen may be administered to the patients with simple face masks using an oxygen flow of around 6-10L/min which may achieve an $\mathrm{FiO}_{2}$ of around 0.5 to 0.6.

This case series has a few limitations, in that, it is an observational study with no randomization, small number of cases and absence of controls. The $\mathrm{FiO}_{2}$ was not measured in the present study, but it was probably around 0.5 to 0.6 with the oxygen flow of around $10 \mathrm{~L} / \mathrm{min}$ using a simple face mask.

\section{Conclusions}

The successful outcome of treatment with supplemental oxygen administration in four cases of pneumothorax of different etiologies has been presented here. Oxygen is a safe, effective and easily available treatment modality which does not require any expertise in its use. It may be helpful in all types of pneumothorax of small size (less than $30 \%$ ) and does not have any serious complications associated with its use. It should be used with caution in patients having a pre-existing chronic lung disease with respiratory failure where its administration may worsen the hypercapnea.

\section{References}

1. Currie G, Alluri R, Christie G, Legge J. Pneumothorax: an update. Postgrad Med J 2007;83:461-5.

2. Sharma A, Jindal P. Principles of diagnosis and management of traumatic pneumothorax. J Emerg Trauma Shock 2008;1:34-41.

3. Northfield T. Oxygen therapy for spontaneous pneumothorax. BMJ 1971;4:86-8.

4. Rhea J, DeLuca S, Greene R. Determining the size of pneumothorax in the upright patient. Radiology 1982;144:733-6.

5. Tschopp J, Bintcliffe O, Astoul P, et al. ERS task force statement: diagnosis and treatment of primary spontaneous pneumothorax. Eur Respir J 2015;46: 32135.

6. Light R. Pneumothorax. In: Light RW, ed. Pleural diseases. 5th Edn. Philadelphia, Lippincott Williams and Wilkins, 2007; pp. 306-39.

7. Collins C, Lopez A, Mathie A, et al. Quantification of pneumothorax size on chest radiographs using interpleural distances: regression analysis based on volume measurements from helical CT. AJR 1995;185:1127-30.

8. Kelly A, Weldon D, Tsang A, Graham C. Comparison between two methods for estimating pneumothorax size from chest X- rays. Resp Med 2006;100:1356-9.

9. MacDuff A, Arnold A, Harvey J. Management of spontaneous pneumothorax: British Thoracic Society pleural disease guideline 2010. Thorax 2010;65:18-31.

10. Parmasivam E, Bodenham A. Airleaks, pneumothorax and chest drains. Continuing education in anaesthesia. Crit Care Pain 2008;8:204-9. 
11. Light R, Lee Y. Pneumothorax, chylothorax, hemothorax, and fibrothorax. In: Mason R, Murray J, Broaddus V, Nadel J, eds. Murray and Nadel's Textbook of Respiratory Medicine. 4th ed. Philadelphia: Elsevier Saunders; 2005. pp. 1961-88.

12. Jindal SK. Oxygen therapy for pulmonary disorders. In: Jindal SK, Agarwal R, editors. Oxygen therapy. 2nd ed. New Delhi: Jaypee; 2008. pp. 157-82.

13. Chadha T, Cohn M. Non invasive treatment of pneumothorax with oxygen inhalation. Respiration 1983;44:147-52.

14. Hill R, DeCarlo D, Hill J, et al. Resolution of experimental pneumothorax in rabbits by oxygen therapy. Ann Thorac Surg 1995;59:825-8.

15. Shih C, Tsai W, Huang S, et al. Effects of high concentration oxygen treatment on traumatic pneumothorax in adult rabbits. Chinese J Physiol 2012;55:17883.

16. Zierold D, Lee S, Subramanian S, DuBois J. Supplemental oxygen improves resolution of injury-induced pneumothorax. J Pediatr Surg 2000;35:998-1001.

17. England G, Hill R, Timberlake G, et al. Resolution of experimental pneumothorax in rabbits by graded oxygen therapy. J Trauma 1998;45:333-4.

18. Shaireen H, Rabi Y, Metcalfe A, et al. Impact of oxygen concentration on time to resolution of spontaneous pneumothorax in term infants: a population based cohort study. BMC Pediatrics 2014;14:208

19. Clark S, Saker F, Schneeberger M, et al. Administration of $100 \%$ oxygen does not hasten resolution of symptomatic spontaneous pneumothorax in neonates.

J Perinatol 2014;34:528-31.

20. Sailaja K, Swetha P. Clinical study of spontaneous pneumothorax. Ind J Appl Res 2015;5:695-7.

21. Havelock T, Teoh R, Laws D, Gleeson F. Pleural procedures and thoracic ultrasound: British Thoracic Society pleural disease guideline 2010. Thorax 2010;65:61-76.

22. Devananda A, Koha M, Onga T, et al. Simple aspiration versus chest-tube insertion in the management of primary spontaneous pneumothorax: a systematic review. Respirat Med 2004;98:57990.

23. McDermott S, Levis D, Arellano R. Chest drainage. Semin Intervent Radiol 2012;29:247-55. 